\title{
Note sulla tradizione del dramma satiresco euripideo in Eustazio
}

Francesco Mori

(University of Roma Tre)

\section{Notes on the tradition of euripidean satyr-plays in Eustathius}

\begin{abstract}
In $12^{\text {th }}$ century some Byzantine scholars claimed to read ancient satyr-plays, but very little evidence of these readings can be found in their erudite works. It has been said that Eustathius read at least one satyr-play, Euripides' Cyclops. He quotes this and other two Euripidean satyr-plays, but an analysis of these quotations seems to hint that he had no direct knowledge of them. Therefore, a critical re-examination of Euripidean quotations in Eustathius is necessary. As a consequence, some points of the history of Euripides' text must also be reconsidered.
\end{abstract}

\section{Keywords}

Euripides; satyr-plays; Byzantine scholars; Eustathius; quotations 
Ł̀ opinione ormai abbastanza radicata fra gli studiosi del teatro antico che all'altezza del XII secolo, nell'ambiente erudito bizantino, fossero note opere appartenenti al genere del dramma satiresco. Tale dato parrebbe deducibile da affermazioni dei filologi del tempo. Giovanni Tzetze, ad esempio, dichiara di leggere drammi satireschi. Si veda

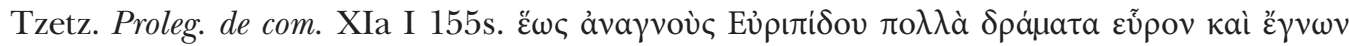

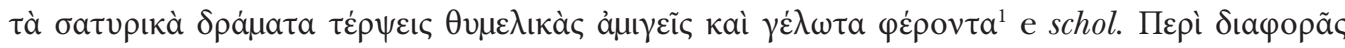

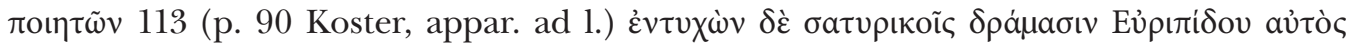

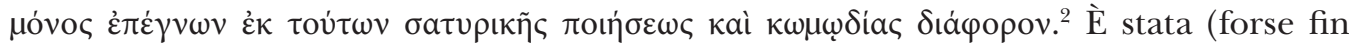
troppo) enfatizzata la propensione di Tzetze a fare sfoggio di erudizione e a vantarsi di aver letto opere pressoché introvabili, ${ }^{3}$ ma è certo che egli aveva accesso a molti materiali oggi perduti. ${ }^{4}$ Purtroppo, le sue asserzioni non sono corroborate da riscontri concreti in quanto non vi sono citazioni satiresche letterali nei suoi scritti, ${ }^{5}$ fatto che di per se stesso non prova che egli non avesse una conoscenza diretta dei drammi satireschi. ${ }^{6}$

Di particolare interesse per lo studio della tradizione del dramma satiresco è il caso di Eustazio di Tessalonica. Con ottimistica fiducia, gli studiosi si sono detti propensi a ritenere che egli leggesse il Ciclope di Euripide, sulla base della nota reperibile nel commento a Hom. Od. XVIII 355 (II p. 184,2-6 Stallbaum):

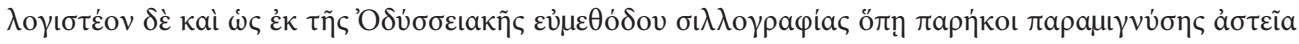

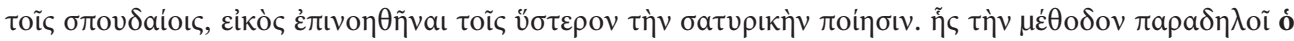

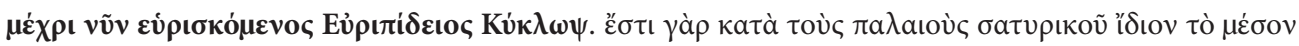

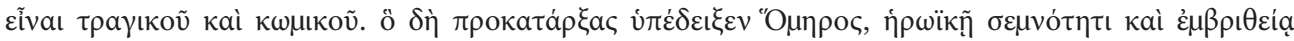

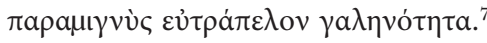

1 "Finché, avendo letto molti drammi di Euripide, ho scoperto e conosciuto i drammi satireschi che ispirano vero piacere teatrale e riso".

2 "Mi sono imbattuto nei drammi satireschi di Euripide e sono stato il solo a poter apprezzare la differenza fra la poesia del dramma satiresco e la commedia”.

$3 \quad$ Vd. Wilson (1990: pp. 297s.; 303).

4 Limitatamente al dramma satiresco euripideo, con apprezzabile cautela, Wilson (1990: p. 303) annota: «egli conosceva, ma sembra ne abbia fatto poco uso, il Ciclope di Euripide, un dramma che non rientrava nella selezione scolastica». Sulla vastità delle letture di Tzetze, vd. Luzzatto (1998: pp. 72-74) e Braccini (2010: p. 88). Luzzatto (1999: pp. 99-102; 159-162) si attesta sulla linea di Masciadri (1987) e di Luppe (1996) nel sostenere che Tzetze leggesse alcune opere euripidee in seguito andate perdute, ed è propensa a credere che anche le affermazioni del filologo sui drammi satireschi siano affidabili.

5 Vd. Koster (1973: p. 408) e Sutton (1988: p. 88, n. 4). Unica eccezione il fr. 372,2s. dell'Euristeo citato in Chil. H. I 516-519 Leone, dedotto da uno scolio a Eur. Hec. 838 ed erroneamente attribuito da Tzetze a Platone comico (vd. Carrara 2021: pp. 210s.).

6 Si è discusso, ad esempio, sull'eventualità che Tzetze leggesse l'Autolico di Euripide, sulla base di Chil. H. VIII 435-453 Leone. Si vedano, a questo proposito, Masciadri (1987), Luppe (1996: pp. 219-221) e Sutton (1988: pp. 90s.).

7 "Bisogna considerare anche il fatto che a partire dalla ben ordinata componente satiresca presente nell'Odissea, che, là dove si attua, fonde insieme elementi spiritosi e seri, verosimilmente i posteri hanno inventato la poesia satiresca. La sua cifra stilistica la chiarisce bene il Ciclope euripideo, ancor oggi disponibile. Infatti, secondo gli antichi, ciò che caratterizza la componente satiresca è il fatto di essere una via di mezzo fra tragico e comico. Omero fu il primo a intraprendere questa strada, mescolando alla solenne gravità della poesia epica un'arguta bonarietà". 
L'espressione impiegata dal Tessalonicese in riferimento all'opera euripidea lascerebbe intendere che essa circolasse ancora o che comunque fosse stata ritrovata di recente. ${ }^{8} \mathrm{Il}$ riferimento di Eustazio potrebbe costituire un riscontro di quanto si legge in Tzetze, ma non siamo in grado di stabilire quanto assidui fossero i rapporti fra i due filologi e se, nel caso in cui uno fosse davvero in possesso di un'opera antica, anche l'altro potesse disporne. ${ }^{9}$ Certo è che il dramma satiresco continuava, evidentemente, a destare l'interesse degli eruditi del XII secolo. Ma, allo stato attuale, l'unico modo per farsi un'idea del grado di conoscenza che uno studioso come Eustazio poteva avere dei drammi satireschi è analizzare i riferimenti e le citazioni presenti nelle sue opere, fermo restando che egli attingeva di frequente ad altri autori, come Ateneo di Naucrati. Pertanto, solo là dove Eustazio si trova a essere unico testimone di un passo, si può ragionevolmente ammettere l'eventualità che leggesse l'opera da cui esso proviene.

Come premessa, si prenda in considerazione il giudizio che l'arcivescovo dà di questo genere letterario in un opuscolo dedicato alla únóкрıбıৎ, dove, dopo aver tessuto l'elogio della tragedia classica, si sofferma sul dramma satiresco e la commedia, dandone una valutazione di ben altro tenore. Si tratta di Eust. $O p$. XIII 6s. (p. 89,35-56 Tafel):

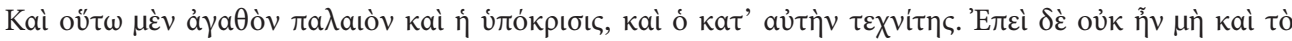

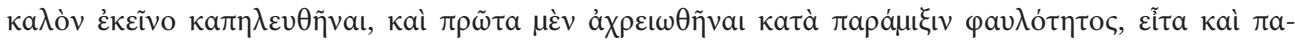

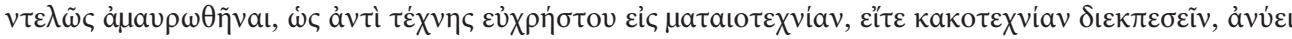

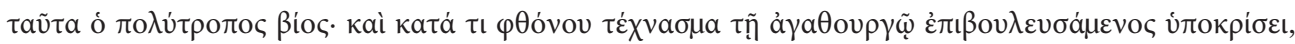

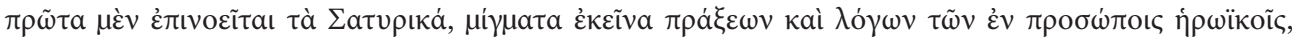

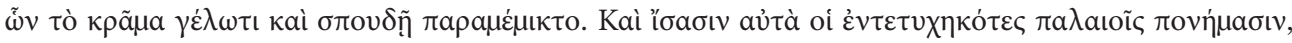

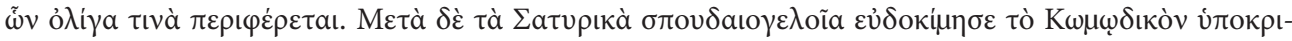

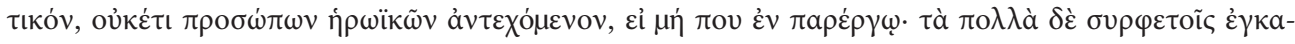

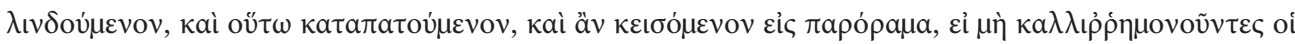

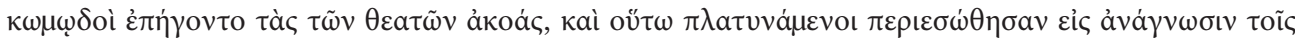

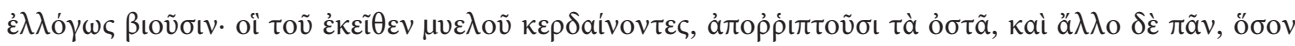

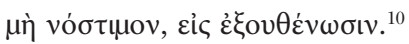

8 "This is the earliest certain reference to the play since antiquity, and suggests both that Cyclops was the only satyr-play known to Eustathius and perhaps that it had only recently been rediscovered": così Hunter \& Laemmle (2020: p. 49). Magnelli (2003) ha ritrovato echi del dramma euripideo in un autore contemporaneo a Eustazio, Teodoro Prodromo.

9 Questo per quanto riguarda i manoscritti degli autori classici. Per un approfondimento sui contatti fra Eustazio e Tzetze, vd. Koster \& Holwerda (1954: pp. 147-152). È tuttavia probabile che leggessero l'uno opere dell'altro, e che, talvolta, si rendessero colpevoli di piccoli furti intellettuali, come ha dimostrato Cullhed (2014: p. 63.).

10 "E così la recitazione era un'eccellenza antica e anche l'artista che la praticava. Ma visto che nemmeno quell'eccellenza poteva mantenersi, ma doveva prima essere contaminata dalla volgarità, poi completamente oscurata, al punto da scadere, da arte utile qual era, in un'attività frivola e di cattivo gusto, l'esistenza perniciosa porta a effetto questi processi: ed escogitando una sorta di invidioso contrappasso ai danni della recitazione virtuosa, ecco che prima inventa i drammi satireschi, quelle commistioni di azioni e parole di personaggi eroici, tenute insieme da un impasto di serio e faceto. Li conoscono bene quanti si sono imbattuti nelle opere letterarie antiche, poche delle quali sopravvivono. Dopo i serio-comici drammi satireschi prevalse un tipo di recitazione comica, che non aveva più come oggetto i personaggi eroici, se non di tanto in tanto, marginalmente; perlopiù si rotola nella volgarità, e vi si crogiola, e sarebbe rimasta 
Si noti che la commistione di serio e faceto, caratteristica del dramma satiresco, incarna, agli occhi di Eustazio, la degenerazione dell'arte teatrale, tanto più che, come si legge anche nel passo dei Commentari omerici summenzionato, lo spirito farsesco investe quegli eroi del mito tradizionale, che erano già voci paradigmatiche della scena tragica. ${ }^{11}$ Ciò detto, non pare che l'aspetto etico abbia influito in modo evidente sulla tradizione dei testi, se si pensa alle numerose citazioni, nell'opera del Tessalonicese, tratte da Aristofane, poeta assai apprezzato nel XII secolo per le sue qualità retoriche e linguistiche. ${ }^{12}$ Da un punto di vista linguistico, infatti, tanto il dramma satiresco quanto la commedia offrivano spunti e raffronti utili al commento dei passi epici. In questa sede, si prenderanno in esame le citazioni tratte da drammi satireschi euripidei presenti in Eustazio, per tentare di comprendere quale fosse il grado delle sue conoscenze in merito.

Cominciamo dall'unico dramma satiresco giunto fino a noi integralmente, il Ciclope di Euripide. Relativamente allo studio sulla presenza di Euripide in Eustazio, il Ciclope ricopre un ruolo fondamentale, perché appartiene, com’è noto, ai drammi cosiddetti "alfabetici", un gruppo di nove opere tramandate in due codici di età medievale, il Laurentianus gr. 32.2 (L) e il Laurentianus conv. soppr. $172+$ Palatinus gr. 287 (P), il rapporto tra i quali costituisce un'annosa vexata quaestio, che non è il caso, in questa sede, di riprendere, nemmeno per sommi capi. Basterà ricordare che il modello da cui i due codici discendono è stato spesso identificato con un esemplare in possesso di Eustazio, o almeno una copia di quell'esemplare. Questo perché, in genere, si è inclini a credere che il Tessalonicese leggesse i drammi "alfabetici" di Euripide. ${ }^{13}$ Allo stato attuale, è bene guardarsi da asserzioni troppo generiche e approcciare la questione in modo più sistematico, ovvero verificare se le citazioni di Eustazio sono di prima mano o se egli dipende da altre fonti identificabili.

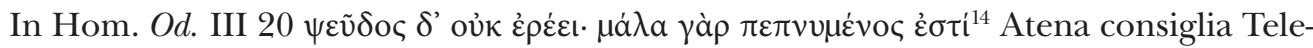
maco di rivolgersi a Nestore per avere notizie del padre, perché Nestore è uomo sincero e avveduto. Eustazio commenta così il passo (I p. 110,43-I p. 111,1 Stallbaum):

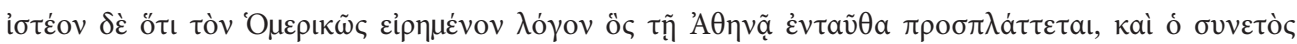

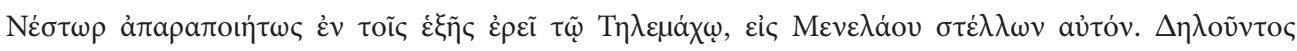

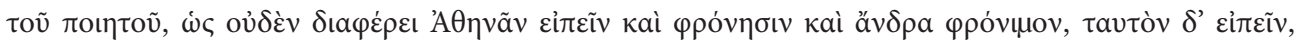

ignorata, non fosse stato che i comici, con il loro linguaggio altisonante, si facevano ascoltare dal pubblico, e così declamandoli fecero sopravvivere questi testi alla lettura delle persone istruite: che tuttavia traggono giovamento dal midollo di quelle rappresentazioni, gettano via le ossa, e tutto il resto, quanto non è commestibile, nei rifiuti”.

11 Per un inquadramento dell'opuscolo di Eustazio, vd. Solarino (1994) e van den Berg (2017: pp. 16-22).

12 A questo proposito, vd. Miller (1942); sugli studi da Eustazio dedicati alla commedia di Aristofane, vd. Wilson (1990: p. 310). Vd., da ultimo, anche van den Berg (2021: pp. 242s.). L'apprezzamento per il poeta

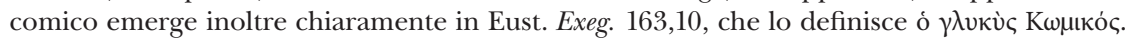

13 Fra le voci più autorevoli che si sono pronunciate in merito si ricordano Zuntz (1955: pp. 146-152), Turyn (1957: pp. 304s.), Pertusi (1957: p. 21), Browning (1960: p. 15), Zuntz (1965: pp. 184s.), Tuilier (1968: pp. 187-209). Alcune osservazioni sono reperibili anche in van der Valk (1971: pp. LXXXVII-XC).

"Non dirà menzogne: è un uomo molto avveduto". 


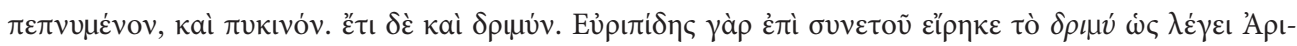

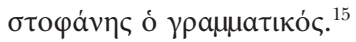

A partire dall'omerico $\pi \varepsilon \pi v v \mu \varepsilon \dot{v o \varsigma}$, l'arcivescovo appronta una dissertazione sui termini che indicano astuzia e avvedutezza, fra cui l'aggettivo $\delta \rho \mu v ́ \varsigma$, che ricorda essere stato usato da Euripide. Sostiene, giustamente, che $\delta \rho \mu v$ c possa essere riferito all'intelligenza

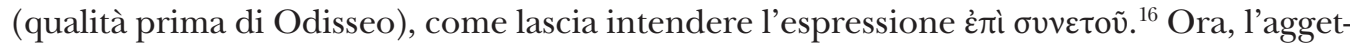
tivo ricorre, nella produzione nota del poeta tragico, solamente in Cycl. 104 oĩ $\delta$ ' äv $\delta \rho a$,

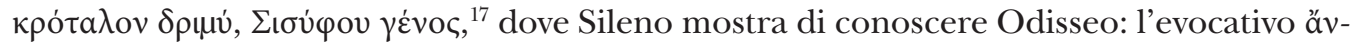

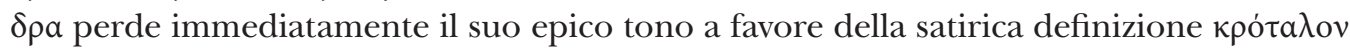
$\delta \rho \mu u^{18}{ }^{18}$ ulteriormente potenziata dall'accenno al fatto che l'eroe sarebbe figlio di Sisifo, personaggio che compare spesso nei drammi satireschi. ${ }^{19}$ Miller (1940: p. 425) annovera, correttamente, Eur. Cycl. 104 fra le allusioni al tragico contenute nell'opera eustaziana. Tuttavia, tale rimando non può deporre a favore di una lettura diretta, da parte di Eustazio, del Ciclope euripideo; non tanto perché il Tessalonicese non ha indicato il titolo dell'opera a cui stava pensando, fatto non insolito per lui, quanto perché dichiara che la

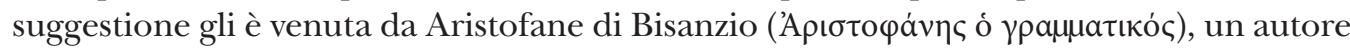
con cui pare avesse una certa familiarità. ${ }^{20}$

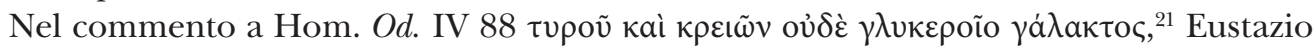
dice (I p. 151,4s. Stallbaum):

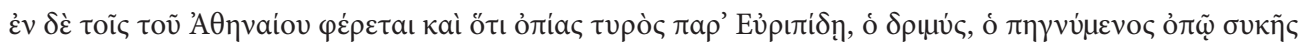

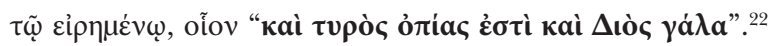

15 "Bisogna sapere che il discorso pronunciato alla maniera omerica, il quale viene qui elaborato da Atena, anche il saggio Nestore lo rivolgerà identico a Telemaco nei versi che seguono, quando lo manderà da Me-

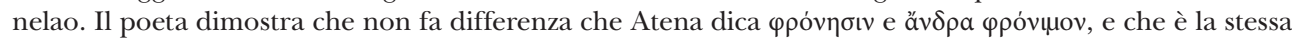

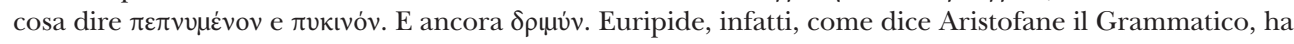

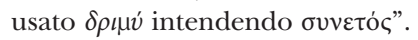

16 L'eroe epico è, per dirla con Biehl (1986: p. 94), uno «scharfmäuliger Radaumacher». Cfr. anche LSJ s.v. $\delta \rho \mu \dot{c}$.

17 "Lo conosco, un petulante sonaglio, figlio di Sisifo".

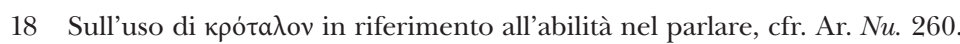

19 A questo riguardo, si vedano le note di Hunter \& Laemmle (2020: pp. 119s.): «Sisyphos was a familiar figure in satyr-play [...]; Aeschylus, Euripides and perhaps Sophocles (fr. 545) all wrote Sisyphos satyr-plays, and Sisyphos also played an important role in at least one of Euripides' satyr-dramas entitled Autolycus. Silenos' view of Odysseus is thus appropriately satyric».

20 Si tratta di Ar. Byz. fr. 31 Slater. Anche altrove Eustazio mostra di conoscere almeno una sezione dell'opera del grammatico, vd., ad esempio, Eust. ad Od. XII 251s. (II p. 22,20-24 Stallbaum). Sulla presenza di Aristofane di Bisanzio nella biblioteca dell'arcivescovo, vd. Wilson (1990: p. 306).

21 "(Né) di formaggio e di carni né di dolce latte".

22 "Nell'opera di Ateno si dice che in Euripide ricorre ỏmías tupóc, il cacio fresco, quello piccante, quello temperato con il cosiddetto succo di fico, come nel passo c'è cacio fresco e c'è latte di Zeus". 


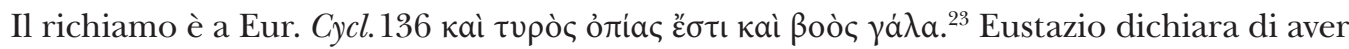
reperito questo passo in un capitolo dei Deipnosofisti, Athen. XIV 658c:

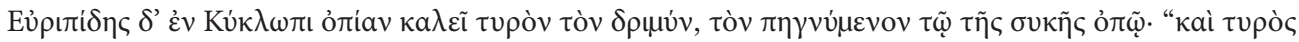

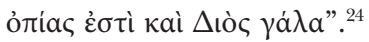

S'aggiunga che questa dipendenza è resa palese anche dalla variante $\Delta$ ı̀̀ (là dove i codici euripidei recano $\beta$ oò $),{ }^{25}$ presente nel testo di Ateneo e in quello di Eustazio. Va da sé che nemmeno questa citazione può essere considerata un indizio attendibile della presenza del Ciclope nella biblioteca del Tessalonicese. È possibile che, quando Eustazio vergava questa sezione del commentario all'Odissea, non avesse a disposizione un manoscritto contenente il dramma satiresco euripideo.

Se da una parte non sorprende che i riferimenti al Ciclope occorrano proprio in questo commentario, per le patenti affinità contenutistiche che accomunano l'opera euripidea e il poema epico, dall'altra si noterà che questi due riferimenti (il primo un'allusione, il secondo una citazione) sono adoperati dall'arcivescovo per incrementare le sue analisi lessicali. Rispetto a questi dati, la testimonianza di Eust. ad Od. XVIII 355 (II p. 184,4

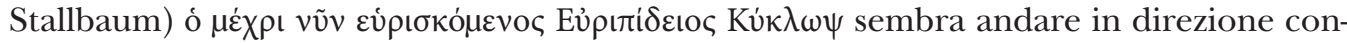
traria e non può mancare di sollevare qualche perplessità. Ci sembra che una possibile spiegazione possa essere la seguente: Eustazio era consapevole che a quell'altezza cronologica il testo euripideo era disponibile e che esso era presente nelle biblioteche di qualche erudito, ma ciò non implica che tale disponibilità si estendesse a lui; inoltre, dal momento che la cronologia relativa delle sue opere è tutt'altro che perspicua, ${ }^{26}$ si può supporre che, quando egli compose alcuni capitoli del commentario odissiaco, non si trovasse più a Costantinopoli e non potesse, di conseguenza, avere accesso a tutti i materiali reperibili nella capitale. ${ }^{27} \mathrm{~A}$ maggior ragione, se si vuole dar credito di attendibilità

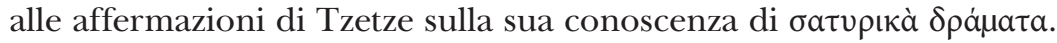

Nell'opera di Eustazio sono reperibili richiami a drammi satireschi euripidei perduti, di cui sopravvivono solo pochi frammenti, perlopiù citati anche da altri autori a lui sicuramente noti. È il caso, ad esempio, dell'Autolico, di cui Athen. X 413c tramanda un lungo passo, il fr. $282 \mathrm{~K}$.:

23 "C'è cacio fresco e c'è latte di vacca".

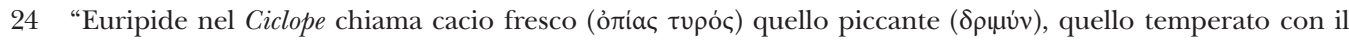
succo di fico: c'è cacio fresco e c'è latte di Zeus".

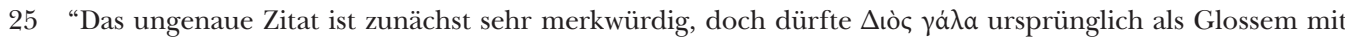
Bezug auf die besondere Wertschätzung der Kuhmilch hinzugefügt und später teilweise als der echte Text angesehen worden sein. Sehr einleuchtend verweist Ussher (S. 60) auf Erasmus, Adagia 1135: quod insigniter esset lautum in cibis, Iovis lac veteres appellabant": così Biehl (1986: p. 99). Contro l'ipotesi di Biehl che $\Delta$ ı̀̀ $\gamma a ́ \lambda \alpha$ sia una glossa, si veda Cipolla (2005: p. 271).

26 Vd. van der Valk (1971: pp. CXXXVII-CXXXIX)

27 A questo proposito, si vedano le osservazioni di Cullhed (2012: p. 447), che richiama l'attenzione sulle aggiunte e le revisioni che Eustazio realizzò, dopo il trasferimento a Tessalonica, sugli autografi delle $\Pi \alpha \rho \varepsilon \kappa \beta 0 \lambda \alpha i$ omeriche, la cui prima redazione risale, com'è noto, al periodo costantinopolitano. 


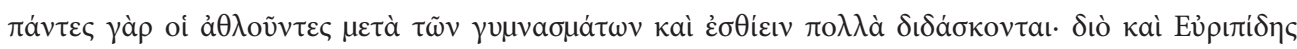

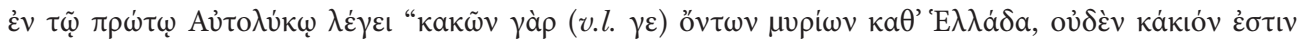

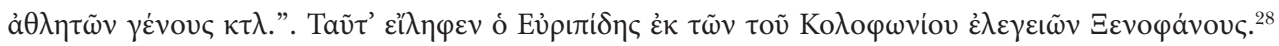

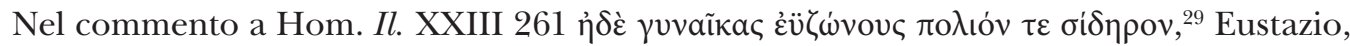
in palese dipendenza da Ateneo, cita i primi due versi del frammento (IV p. 723,6-11 Valk):

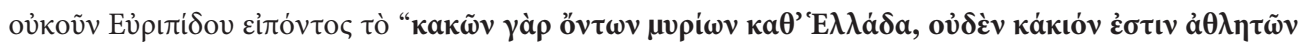

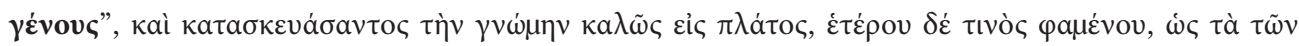

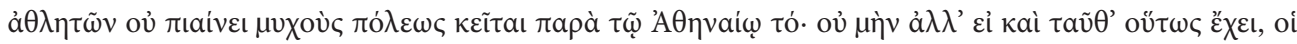

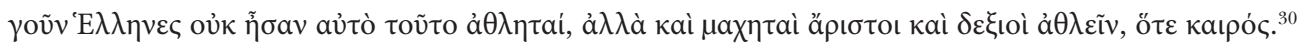

L'occorrenza non sembra sufficiente per indicare che Eustazio avesse una conoscenza diretta di questo dramma satiresco.

Più interessante è senz'altro il caso del Sileo di Euripide, un dramma satiresco sulla cui trama siamo sufficientemente ragguagliati da diversi testimoni, ${ }^{31}$ oltre che da due malconci frustoli papiracei che hanno tramandato i resti di una hypothesis dell'opera. ${ }^{32} \mathrm{Il}$ frammento che interessa prendere in esame in questa sede è il $693 \mathrm{~K}$. $\langle x-\cup-x>$ Ẽ̃a

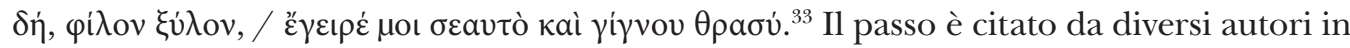
merito alla facies linguistica. Così è per Ap. Dysc. Pron. 140 (p. 73,14-17 Schneider), che cita il secondo verso come esempio di un uso anomalo del riflessivo in caso accusativo:

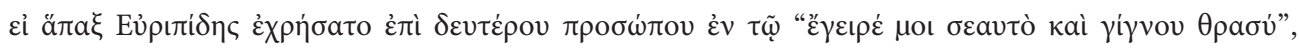

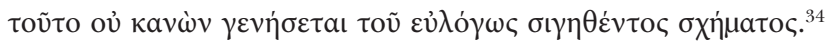

Similmente, Iohann. Philop. Praec. ton. 113 Xenis:

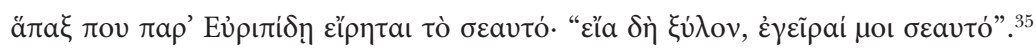

28 "Tutti gli atleti, infatti, con gli esercizi vengono educati anche a mangiare molto; perciò Euripide, nel primo Autolico, dice: moltissime le stirpi malvagie in Grecia, ma nessuna è peggio della stirpe degli atleti. Questo concetto Euripide lo ha desunto dalle elegie di Senofane di Colofone".

29 "Donne dalla bella cintura e ferro grigio".

30 "Dunque, Euripide ha detto molte le stirpi malvagie in Grecia, ma nessuna è peggio della stirpe degli atleti, e ha ben organizzato il pensiero in generale, mentre un altro ha detto che le azioni degli atleti non rimpinguano le casse dello stato, e in Ateneo si legge: nondimeno, se le cose stanno cosi, in ogni caso $i$ Greci non erano proprio per questo atleti, ma erano eccellenti combattenti e abili lottatori, quando si presentava l'occasione".

31 Sulla vicenda di Sileo e sulla distruzione delle sue proprietà da parte di Eracle, si vedano [Apollod.] II 6,2-3, Diod. IV 31,7 e Tzetz. Proleg. de com. XIa II 59-70. Vd. inoltre Tzetz. Chil. H. II 412-438 Leone.

32 Si tratta di P.Strasb. 2676 fr. A e di P.Oxy. XXVI 2455 fr. 8.

33 " $<x-\cup-x>$ suvvia, o mia clava, / destati e sii ardita".

34 "Se in una sola occasione Euripide se ne è servito alla seconda persona nel verso svegliati e sii ardito, non sarà questa una regola della forma ragionevolmente passata sotto silenzio".

35 "Da qualche parte in Euripide si trova, una sola volta, oeavtó: Suvvia clava, destati". 
Il Filopono parrebbe dipendere da Apollonio Discolo, ma resta il fatto che il Filopono cita parte del primo e parte del secondo verso, mentre Apollonio solo il secondo per intero. Il frammento satiresco è tramandato anche da Et. Gen. (B) s.v. čla nella forma oĩa

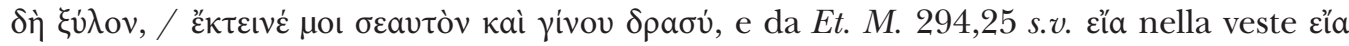

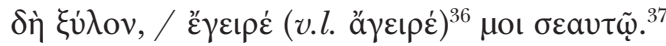

La testimonianza di Eustazio presenta alcuni aspetti di interesse. L'arcivescovo cita

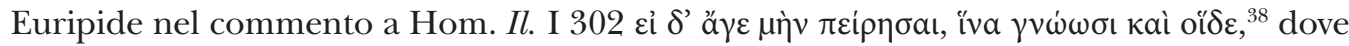
elabora questa spiegazione (I p. 167,30-168,1 Valk):

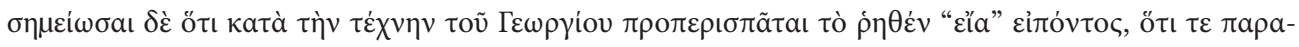

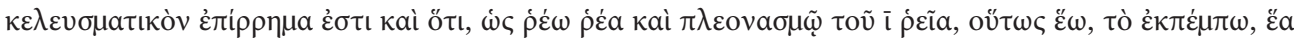

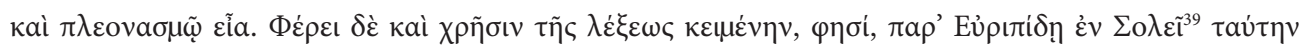

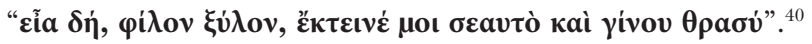

Il suo interesse verte sulle locuzioni esortative e sulla presunta doppia accentazione di عĩa. Lo spunto per queste osservazioni parrebbe venire a Eustazio dalla lettura dell'ope-

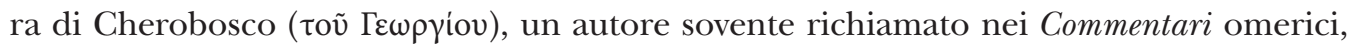

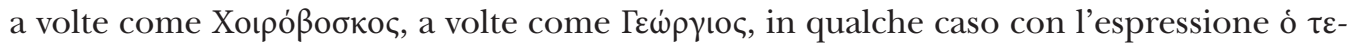
$\chi v \iota \kappa o ́ c .{ }^{41}$ Tuttavia, in ciò che resta dell'opera di questo erudito non si trovano riferimenti al frammento euripideo. D'altra parte, anche Et. Gen. (B) s.v. Ela cita Cherobosco come fonte. È possibile che la sezione dell'opera di Cherobosco in cui compariva la citazione del Sileo sia scomparsa dalla circolazione dopo il periodo di attività di Eustazio. Sotto il profilo testuale, inoltre, è da notare che Eustazio condivide con l'Etymologicum genuinum

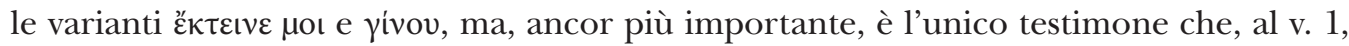
tramanda $\varphi$ í $\mathrm{ov}^{42}$ e che indica il titolo dell'opera da cui i versi provengono.

Alcuni studiosi hanno ravvisato nel passo un senso osceno. $\grave{E}$ infatti probabile che la persona loquens del nostro frammento sia Eracle e che esso vada collocato in un momen-

36 La lettura äjeı siderazione una possibile eco dell'espressione euripidea in Suet. Ner. 49: interdum segnitiem suam his verbis

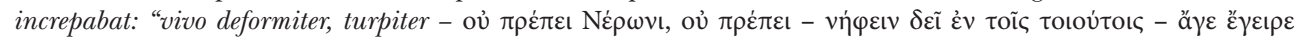

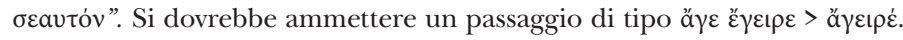

37 È stato convincentemente sostenuto da Cipolla (2017: p. 229) che, in origine, i grammatici che si interes-

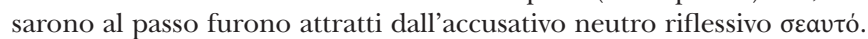

38 "E allora provaci, affinché anche costoro sappiano".

39 Si tratta senza dubbio di un errore del manoscritto laurenziano, da correggere $\Sigma$ $v \varepsilon \varepsilon i$ sulla scorta di van der Valk (1971: p. 167).

40 "Nota che ela è properispomeno secondo la grammatica di Cherobosco, il quale dice che si tratta di un

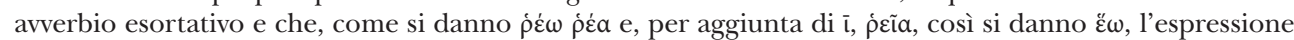

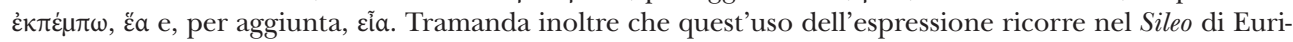
pide: Suvvia, mia clava, tenditi e sii ardita".

41 Lo stesso epiteto, come non manca di sottolineare van der Valk (1971: p. LXXIII, n. 1), viene attribuito anche ad Erodiano. Sulla presenza di Cherobosco in Eustazio, vd. anche Erbse (1950: p. 3).

42 Che fu eliminato da Nauck (1889: p. 577). 
to di festosità triviale, conseguente alla distruzione delle proprietà di Sileo. ${ }^{43}$ Eracle si rivolgerebbe al proprio membro virile. Tale lettura parrebbe trovare supporto in Hesych.

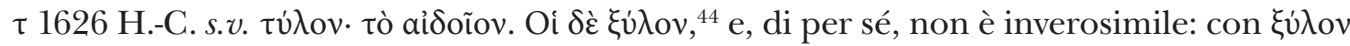
Eracle, rivolgendosi alla figlia di Sileo, Senodoce, nel tentativo di sedurla, attiverebbe un gioco di parole che coinvolge il proprio membro e la clava. ${ }^{45}$ Peraltro, l'espressione

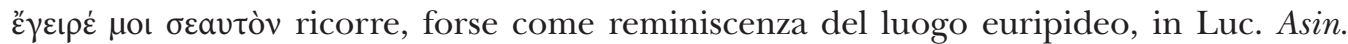
5 , una triviale scena di seduzione, che potrebbe supportare l'interpretazione oscena del frammento. ${ }^{46}$ Stando così le cose, da un punto di vista metrico, linguistico e semantico,

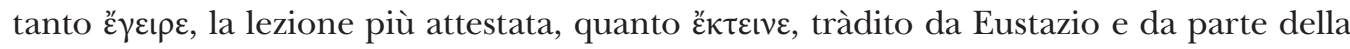

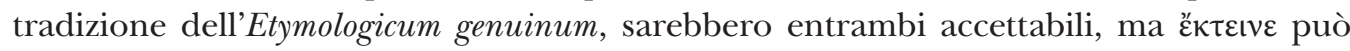
essere una spiegazione di ع̌ $\gamma \varepsilon\llcorner\rho \varepsilon$ erroneamente confluita nel testo, a conferma, però, dell'interpretazione di cui sopra. ${ }^{47}$

Per riassumere, si può osservare che la testimonianza di Eustazio è preziosa, in primo luogo, perché ha preservato il passo di Euripide nella veste più completa; in secondo luogo, perché le varianti che caratterizzano la sua citazione potrebbero influire sulla lettura di questi versi; da ultimo, perché grazie a lui conosciamo il titolo dell'opera da cui è stato tratto il passo. Ma, ancora una volta, il fatto che Eustazio dichiari di aver reperito la citazione in un'altra fonte, nella fattispecie Cherobosco, non depone a favore di una lettura diretta dell'opera.

Dalle occorrenze che sono state prese in esame nel corso della trattazione non sembra possibile dedurre che Eustazio possedesse una conoscenza diretta di drammi satireschi euripidei, ovvero che leggesse molto più di quanto traspaia dai suoi scritti. Per quanto pertiene il Ciclope, le osservazioni svolte non inficiano in toto l'ipotesi che il Tessalonicese leggesse i drammi "alfabetici" di Euripide, ma suggeriscono, più ragionevolmente, che egli fosse in possesso di (o poté consultare) un manoscritto contenente un certo numero di opere euripidee, probabilmente non tutte quelle che oggi leggiamo, bensì solo una parte. Come già Zuntz (1955: p. 148, n. 1) aveva rilevato, i riscontri presenti negli scritti di Eustazio non sembrano, di per sé, assurgere a indizi di una lettura diretta del Ciclope. ${ }^{48}$

43 Vd. Jouan \& Van Looy (2002: p. 83).

44 La lemmatizzazione all'accusativo suggerisce che quella di Esichio sia una citazione letteraria, forse comi-

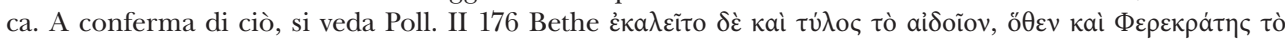

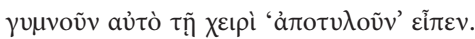

45 Gli argomenti di Pechstein (1998: pp. 269s.) contro questa interpretazione si presentano poco solidi, lo studioso si limita a rilevare che per $\xi u ́ \lambda o \varsigma$ non è ammissibile il senso di "fallo". Poco convincenti anche le ragioni addotte dallo stesso (p. 270) contro l'identificazione con Eracle del personaggio che pronuncia queste parole: Eracle, che altrove invita coraggiosamente Sileo a bere con lui (fr. 691 K.), non avrebbe bisogno di ricorrere a una tale forma di incoraggiamento. Contro l'interpretazione oscena, vd. anche Cipolla (2017: l.c.), che pensa piuttosto a una sorta di formula magica con cui il personaggio ordinerebbe alla clava di sollevarsi. A sostegno di questa lettura, lo studioso rimanda a Eur. Cycl. 646-648, dove si parla di una formula magica per far muovere da solo il tronco con cui i Greci accecheranno Polifemo.

46 Così ha osservato, giustamente, Cipolla (2017: pp. 241s.).

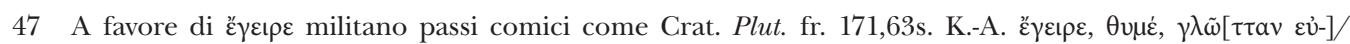

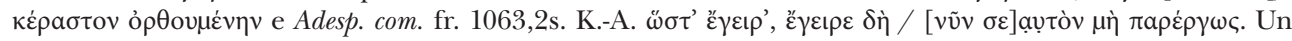
influsso comico sul dramma satiresco euripideo è tutt'altro che inverosimile.

48 Lo studioso tuttavia, com'è noto, sostiene la teoria per cui il capostipite di $\mathbf{L}$, almeno per quanto pertiene 
Quanto ai drammi satireschi frammentari, nonostante le affermazioni di Tzetze (vd. supra), non sembra che Eustazio consultasse gli stessi volumi del suo collega. Le poche citazioni che reperiamo nei Commentari omerici confermano piuttosto una dipendenza del Tessalonicese da altre fonti.

Ciò nonostante, dalla lettura di Eustazio ricaviamo la conferma che, per quanto la tradizione testuale sia stata assai meno generosa con il dramma satiresco che con la tragedia, questo genere letterario destava un certo interesse ancora negli ambienti eruditi bizantini del XII secolo, soprattutto sotto il profilo linguistico. Nel caso di Eustazio, inoltre, esso si presentava particolarmente adatto a incrementare il commento dei poemi omerici, non solo per via della lingua, ma anche in virtù dei contenuti, dal momento che gli stessi eroi del mito, oggetto dei racconti di Omero, popolavano, al tempo di Euripide, la scena teatrale.

\section{Bibliografia}

Biehl, W. (1986). Euripides. Kyklops. Heidelberg: C. Winter Universitätsverlag.

Braccini, T. (2010). Mitografia e miturgia femminile a Bisanzio: il caso di Giovanni Tzetze. I Quaderni del Ramo d'Oro on-line, 3, 88-105.

Browning, R. (1960). Recentiores non deteriores. Bulletin of the Institute of Classical Studies, 7, 11-21. Carrara, L. (2021). Giovanni Tzetze, il dramma satiresco ed il Fortleben di Euripide a Bisanzio: nuove letture di vecchi testimoni. Medioevo greco, 21, 171-214.

Cipolla, P. (2005). Su alcune citazioni euripidee in Ateneo. Prometheus, 31, 263-281.

Cipolla, P. (2017). Il dramma satiresco e l'erudizione antica: sull'uso delle citazioni satiresche nelle fonti di tradizione indiretta. Lexis, 35, 221-248.

Cullhed, E. (2012). The Autograph Manuscripts Containing Eustathius' Commentary on the Odyssey. Mnemosyne, 65, 445-461.

Cullhed, E. (2014). The blind bard and 'I': Homeric biography and authorial personas in the twelfth century. Byzantine and Modern Greek Studies, 38(1), 49-67.

Erbse, H. (1950). Untersuchungen zu den attizistischen Lexika. Berlin: Akademie Verlag.

Hunter, R., \& Laemmle, R. (2020). Euripides. Cyclops. Cambridge: Cambridge University Press.

Jouan, F., \& Van Looy, H. (2002). Euripide. Tragédies, VIII: Fragments (Sthénébée-Chrysippos). Paris: Les belles lettres.

Kannicht, R. (2005). Tragicorum graecorum fragmenta, V/1: Euripides. Göttingen: Vandenhoeck \& Ruprecht.

Koster, W. J. W. (Rec.). (1973). M. van der Valk, Eustathii Archiepiscopi Thessalonicensis Commentarii ad Homeri Iliadem pertinentes. Vol. I: Commentarios ad libros A- $\Delta$ complectens. Leiden, Brill, 1971. Mnemosyne, 26, 404-411.

i drammi "alfabetici", sarebbe stato un manoscritto in possesso di Eustazio, o una copia di questo (Zuntz 1955: pp. 151s.). Ciò presuppone che, dal momento che $\mathbf{L}$ contiene tutti i drammi esclusi dalla selezione, anche tale esemplare li tramandasse, senonché, dalle citazioni presenti in Eustazio, pare si possa desumere che di molti di quei testi egli avesse solo una conoscenza indiretta. Questa circostanza potrebbe di per sé revocare in dubbio l'esatta identificazione del modello di $\mathbf{L}$ con la copia di Eustazio. 
Koster, W. J. W., \& Holwerda, D. (1954). De Eustathio, Tzetza, Moschopulo, Planude Aristophanis commentatoribus. Mnemosyne, 7, 136-156.

Leone, P. A. (1968). Ioannis Tzetzae Historiae. Napoli: Libreria scientifica editrice.

Luppe, W. (1996). Zur ,Lebensdauer‘ der Euripides-Hypotheseis. Philologus, 140, 214-224.

Luzzatto, M. J. (1998). Leggere i classici nella biblioteca imperiale: note tzetziane su antichi codici. Quaderni di storia, 48, 69-86.

Luzzatto, M. J. (1999). Tzetzes lettore di Tucidide. Note autografe sul codice Heidelberg Palatino Greco 252. Bari: Dedalo.

Magnelli, E. (2003). Un nuovo indizio (e alcune precisazioni) sui 'drammi alfabetici' di Euripide a Bisanzio tra XI e XII secolo. Prometheus, 29, 193-212.

Masciadri, V. (1987). Autolykos und der Silen. Eine übersehene Szene des Euripides bei Tzetzes. Museum Helveticum, 44, 1-7.

Miller, H. (1940). Euripides and Eustathius. American Journal of Philology, 61(4), 422-428.

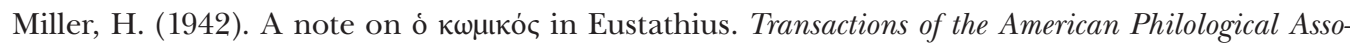
ciation, 73, 353-357.

Nauck, A. (1889²). Tragicorum graecorum fragmenta. Lipsiae: B. G. Teubneri.

Pechstein, N. (1998). Euripides Satyrographos. Ein Kommentar zu den Euripideischen Satyrspielfragmenten. Stuttgart - Leipzig: B. G. Teubner.

Pertusi, A. (1957). Selezione teatrale e scelta erudita nella tradizione del testo di Euripide, III. La ricomparsa di Euripide nel Medio Evo bizantino. Dioniso, 20, 18-37.

Solarino, М. (1994). Il ПЕРI ҮПОКРІ $\mathrm{E} \Omega \Sigma$ di Eustazio di Tessalonica. Giornale italiano di filologia, 46, 277-290.

Stallbaum, J. G. (1825-1826). Eustathii archiepiscopi Thessalonicensis Commentarii ad Homeri Odysseam ad fidem exempli romani editi (vols. I-II). Lipsiae: Weigel.

Sutton, D. F. (1988). Evidence for lost dramatic hypotheses. Greek, Roman and Byzantine Studies, $29,87-92$.

Tafel, T. L. F. (1832). Eustathii Metropolitae Thessalonicensis Opuscula (accedunt Trapezuntinae historiae scriptores Panaretus et Eugenicus). Francofurti ad Moenum: Sigismundi Schmerber.

Tuilier, A. (1968). Recherches critiques sur la tradition du texte d'Euripide. Paris: Librairie C. Klincksieck.

Turyn, A. (1957). The Byzantine manuscript tradition of the tragedies of Euripides. Urbana: The University of Illinois Press.

Van den Berg, B. (2017). 'The Excellent Man Lies Sometimes': Eustathios of Thessalonike on Good Hypocrisy, Praiseworthy Falsehood, and Rhetorical Plausibility in Ancient Poetry. Scandinavian Journal of Byzantine and Modern Greek Studies, 3, 15-35.

Van den Berg, B. (2021). Playwright, Satirist, Atticist: The Reception of Aristophanes in 12th-Century Byzantium. In P. Marciniak, \& I. Nilsson (Eds.), Satire in the Middle Byzantine Period. The Golden Age of Laughter? (Vol. 12; pp. 227-253). Leiden - Boston: Brill.

Van der Valk, M. (1971-1987). Eustathii archiepiscopi Thessalonicensis Commentarii ad Homeri Iliadem pertinentes, ad fidem codicis Laurentiani editi (vols. I-IV). Lugduni Batavorum: Brill.

Wilson, N. (1990). Filologi bizantini (Trad. it. di G. Gigante). Napoli: Morano Editore.

Zuntz, G. (1955). The political plays of Euripides. Manchester: Manchester University Press. 
Francesco Mori

Note sulla tradizione del dramma satiresco euripideo in Eustazio

Zuntz, G. (1965). An Inquiry into the Transmission of the Plays of Euripides. Cambridge: Cambridge University Press.

Francesco Mori / francesco.mori@uniroma3.it

PhD candidate

Department of Humanities

University of Roma Tre

Via Ostiense, 159, 00154 Roma, Italy

This work can be used in accordance with the Creative Commons BY-SA 4.0 International license terms and conditions (https://creativecommons.org/licenses/by-sa/4.0/legalcode). This does not apply to works or elements (such as image or photographs) that are used in the work under a contractual license or exception or limitation to relevant rights 